\begin{tabular}{|c|c|}
\hline Title & CRISPRa mediated NEA T1 IncRNA upregulation induces formation of intact paraspeckles \\
\hline Author(s) & Y amazaki, Tomohiro; Fujikawa, Chikako; Kubota, A yaka; Takahashi, A kinari; Hirose, Tetsuro \\
\hline Citation & $\begin{array}{l}\text { Biochemical and biophysical research communications, 504(1), } 218.224 \\
\text { https://doi.org/10.1016j.bbrc.2018.08.158 }\end{array}$ \\
\hline Issue Date & $2018-09-26$ \\
\hline Doc URL & http:/hdl .handle.net/2115/75519 \\
\hline Rights & $\begin{array}{l}\text { @ 2018. This manuscript version is made available under the CC-BY-NC-ND } 4.0 \text { license } \\
\text { http://creativecommons.org/icenses/by-nc-nd/4.0/ }\end{array}$ \\
\hline Rights(URL) & http://creativecommons.org/icenses/by-nc-nd/4.0/ \\
\hline Type & article (author version) \\
\hline File Information & BBRC-S-18-10512-2.pdf \\
\hline
\end{tabular}

Instructions for use 


\section{CRISPRa-mediated NEAT1 IncRNA upregulation induces formation of intact paraspeckles}

Tomohiro Yamazaki ${ }^{\mathrm{a}, \mathrm{b}}$, Chikako Fujikawa ${ }^{\mathrm{a}}$, Ayaka Kubota ${ }^{\mathrm{a}}$, Akinari Takahashi, ${ }^{\mathrm{a}, \mathrm{b}}$, Tetsuro Hirose $e^{a, b^{*}}$

anstitute for Genetic Medicine, Hokkaido University, Sapporo 060-0815, Japan

${ }^{\mathrm{b}}$ Graduate School of Medicine, Hokkaido University, Sapporo 060-8638, Japan

${ }^{*}$ Corresponding author. Institute for Genetic Medicine, Hokkaido University, Kita 15 Nishi 7, Kita-ku, Sapporo 060-0815, Japan

E-mail address: hirose@igm.hokudai.ac.jp 


\section{Abstract}

Long noncoding RNAs (IncRNAs) are fundamental genomic regulatory factors under various physiological and pathological conditions. A class of IncRNAs termed architectural RNAs (arcRNAs) plays an essential scaffolding role in building nuclear bodies. NEAT1 arcRNA is an abundant, nuclear-retained IncRNA that constructs paraspeckle nuclear bodies. NEAT1 is upregulated in various developmental and disease conditions including cancer and virus infection. However, it remains unclear how elevated expression of NEAT1 influences such conditions. Here, we set up an experimental method to selectively increase NEAT1 expression. We applied the synergistic activation mediator (SAM) system using catalytically dead Cas9 (dCas9) proteins to activate transcription of the NEAT1 gene. We examined 10 pre-designed and 15 originally designed single-guide RNAs (sgRNAs) in the NEAT1 promoter region for CRISPR activation (CRISPRa). We validated several sgRNAs that we designed for the SAM system to strongly activate NEAT1 expression in two human cell lines and induced formation of paraspeckles with intact core-shell structures. Thus, this selective NEAT1 upregulation method using the SAM system would be useful for further functional analyses of NEAT1 IncRNA in both basic and applied research.

Keywords: CRISPRa, dCas9, Long noncoding RNA, NEAT1, Paraspeckle, Nuclear body 


\section{Introduction}

Mammalian genomes are pervasively transcribed and produce tens of thousands of long noncoding RNAs (IncRNAs), which lack protein-coding potential and are greater than 200 nucleotides in length [1]. Functional and mechanistic studies over the last decade have shown that IncRNAs are fundamental regulators for various cellular processes such as gene expression and three-dimensional (3D) nuclear organization, and play regulatory roles under physiological and pathological conditions [2, 3]. LncRNAs themselves can be typically functional molecules by forming complexes with RNA-binding proteins and/or other RNAs. Mechanistically, IncRNAs can act as guides, scaffolds, decoys, or enhancers [3, 4]. Some nuclear IncRNAs affect expression of nearby genes in cis and some can regulate distal chromosomal loci in trans [3]. As the nuclear IncRNAs carry positional information in the nucleus, they can perform these functions by transcription from their endogenous chromosomal loci $[3,5]$. Thus, to understand the functions and molecular mechanisms, it would be important to investigate the IncRNAs expressed from original loci.

A class of nuclear IncRNAs called architectural RNAs (arcRNAs) plays an architectural role in construction of membraneless subnuclear bodies [6]. NEAT1 is the arcRNA that constructs paraspeckles, nuclear bodies found in close proximity to nuclear speckles [7-12]. The NEAT1 gene generates two isoforms of NEAT1 transcripts, NEAT1_1 ( 3.7 kb in human) and NEAT1_2 ( 22.7 kb in human), by alternative 3' end processing [13]. NEAT1_2 is essential for paraspeckle formation, while NEAT1_1 is dispensable [13]. The paraspeckle is composed of more than 60 kinds of paraspeckle proteins (PSPs) as well as NEAT1 IncRNAs [13-15]. The paraspeckle is co-transcriptionally formed near the transcription site of NEAT1. Interestingly, the paraspeckle possesses a highly ordered structure. An electron microscopic study revealed that NEAT1 5' and 3' regions are located in the shells of paraspeckles, and the NEAT1 middle region is located in the core of paraspeckles [16]. In addition, super-resolution microscopic analyses showed core-shell spheroidal paraspeckle structures [17]. Such a paraspeckle sphere contains about 50 NEAT1_2 molecules [18]. Most recently, we found modular RNA domains in NEAT1 IncRNA. The NEAT1 middle domain is essential for paraspeckle assembly, consistent with the core localization of this region [19]. The core also contains multiple PSPs essential for paraspeckle formation (e.g., FUS) that possess prion-like domains as aggregate-prone protein domains [20]. Additionally, paraspeckles have a dynamic nature in which the PSPs go in and out of the body [21]. These characteristics are consistent with the idea that the paraspeckle is a phase-separated nuclear structure [10, 19]. 
Functionally, the paraspeckles act as molecular sponges to sequestrate specific RNAs and proteins to regulate freely available pools of these molecules, leading to changes in gene expression [20, 22-24]. NEAT1 binds hundreds of active chromatin sites, suggesting a role in gene regulation and chromosome organization [25]. Furthermore, recent studies showed that NEAT1 is involved in development and disease including cancer [26-29]. Although many physiological functions of NEAT1 have been reported, it remains unclear how NEAT1 upregulation influences cellular events. So far, expression of full-length human NEAT1_2 in cells from plasmids has not been reported. Furthermore, paraspeckles are co-transcriptionally formed, suggesting the importance of the spatial position of the NEAT1 gene within the nucleus for its precise functions. Recent technology using clustered regulatory interspaced short palindromic repeats (CRISPR)/Cas9 called CRISPR activation (CRISPRa) can selectively activate a gene of interest from its original chromosomal locus [30]. One of the technologies is a synergistic activation mediator (SAM), which selectively and robustly activates transcription [31]. This technology was applied to genome-wide activation of protein-coding and noncoding genes to search for their biological roles [31, 32]. This SAM system requires catalytically dead Cas9 (dCas9) protein fused to the VP64 transcriptional activator and single-guide RNA (sgRNA) containing tandem MS2 aptamers to recruit additional transcriptional activator complexes, MS2-P65-HSF1 fusion proteins [31].

Here, we demonstrated selective activation of NEAT1 transcription by the SAM system. We observed robust transcriptional activation of NEAT1 in several human cell lines. We also confirmed that the effective sgRNAs can induce formation of core-shell paraspeckles. The method established here would be one of the platforms to investigate molecular functions and roles of paraspeckles constructed by NEAT1 IncRNAs in gene expression and 3D genome organization. 


\section{Materials and Methods}

\subsection{Cell culture}

HAP1 cells (Horizon Discovery) were maintained in IMDM (Gibco) supplemented with 10\% FBS (Gibco) and Penicillin-Streptomycin (Nacalai Tesque). U2OS cells were maintained in DMEM (Gibco) containing 10\% FBS (Gibco) and Penicillin-Streptomycin (Nacalai Tesque). MG132 (Sigma-Aldrich, $5 \mu \mathrm{M}$ for 6 hours) was used to enhance NEAT1 expression [22].

\subsection{Plasmid construction}

The plasmids (sgRNA(MS2) cloning backbone [Plasmid \#61424], dCAS9-VP64_GFP [Plasmid \#61422], and MS2-P65-HSF1_GFP [Plasmid \#61423]) were both obtained from Addgene, gifted by, Feng Zhang. sgRNAs targeting NEAT1 promoter regions were designed using the CRISPR design website (http://crispr.mit.edu/), and their scores for faithfulness of on-target activity were calculated using the same website. Sequence data of sgRNA(MS2) library were obtained from a published dataset [32]. The sgRNAs were cloned into sgRNA(MS2) cloning backbone plasmids at the Bbsl site according to a previous study [33].

\subsection{Plasmid transfection}

All plasmids for transfection experiments were purified with QIAGEN Plasmid Midi Kit (QIAGEN). To introduce plasmids for the SAM system into HAP1 and U2OS cells, $4 \mu \mathrm{g}$ (HAP1) or $2 \mu \mathrm{g}$ (U2OS) of each plasmid (sgRNA(MS2) cloning backbone, dCAS9-VP64_GFP, and MS2-P65-HSF1_GFP) was transfected into HAP1 cells $\left(3 \times 10^{6}\right.$ cells $)$ or U2OS cells $\left(1 \times 10^{6}\right.$ cells) using Nucleofector Kit $\mathrm{V}$ (Lonza) with a Nucleofector device (Lonza) using program "X-005" or "X-001", respectively, according to the manufacturer's instructions. Transfection efficiency was determined by counting GFP-positive cells with Volocity (PerkinElmer) using auto settings.

\subsection{Reverse transcription-quantitative PCR (RT-qPCR)}

Total RNAs were purified from cells with TRI reagent (Molecular Research Center) as previously described [19]. The total RNAs $(1 \mu \mathrm{g})$ were reverse-transcribed using the High Capacity cDNA Reverse Transcription Kit (Thermo Fisher Scientific) with random hexamer primers. Aliquots of cDNA were amplified by qPCR using LightCycler 480 SYBR Green I Master reagent (Roche) according to the manufacturer's instructions. 18S rRNA levels were used for normalization. Primers used in this analysis are listed in Table S1. 


\subsection{Single molecule FISH (smFISH)}

Pre-designed Stellaris FISH probes to the NEAT1 middle region (LGC Biosearch Technologies) were used. The smFISH was performed according to the manufacturer's instructions as described previously [19]. The images were captured with confocal microscope FV1000 (Olympus). For quantification, the images were analyzed with Volocity. Detection of GFP-positive cells and DAPI signals was performed with Volocity using the auto settings. NEAT1 smFISH signals were detected with an intensity threshold. Using the obtained quantified data, graphing and statistical analysis were performed with Prism 7 (GraphPad Software).

\subsection{Structured illumination microscopy (SIM)}

SIM observation was performed as described [19]. Briefly, digoxigenin (DIG)-labeled NEAT1 probes to the 5 ' end of NEAT1 $(+1$ to +1000$)$ and the 3 ' end of NEAT1_2 $(+21743$ to +22580$)$ were synthesized using T7 or SP6 RNA polymerase and a DIG RNA labeling kit (Roche) as described [19]. The synthesized probes for NEAT1 5' and 3' ends were used as mixtures to detect shells of paraspeckles. The DIG-labeled probes were detected by anti-DIG monoclonal antibody (Abcam, 21H8, ab420, 1:100 dilution) and anti-mouse secondary antibody conjugated with Cy3 (Millipore, AP124C, 1:100 dilution). GFP-positive cells were visualized by anti-GFP rabbit polyclonal antibody (MBL, 1:100 dilution) and anti-rabbit secondary antibody conjugated with Cy2 (Abcam, ab6940, 1:100 dilution). SIM images were captured using ELYRA PS.1 (ZEISS) with $100 \times$ objective lens as described [19]. 


\section{Results}

\subsection{CRISPR/Cas9 SAM can specifically induce NEAT1 IncRNA in human cell lines}

To selectively activate transcription of the NEAT1 gene, we employed the SAM system, which has been used in many studies including genome-wide screens [31] (Figure 1A). For NEAT1 transcriptional activation, we tested 25 sgRNAs including 10 pre-designed sgRNAs from the SAM sgRNA library and 15 originally designed sgRNAs [32] (Table 1). The 10 pre-designed sgRNAs target broadly located positions of the NEAT1 promoter $(-700$ to +1$)$ (Figure 1B). Our 15 original sgRNAs target a more proximal promoter region $(-200$ to +1$)$, where the highest levels of SAM-mediated activation of multiple genes were achieved in a previous study [31] (Figure 1B). These include sgRNAs with sense and antisense directions to the promoter (Figure 1B). We first examined the SAM system in HAP1 cells and evaluated the effect on NEAT1 expression by monitoring the expression levels at the 5 ' terminal region, where NEAT1_1 and NEAT1_2 overlap, and the NEAT1_2 specific region using RT-qPCR (Figure 1C). Although some sgRNAs such as Lib\#10, \#11, \#12,\#14, and \#15 did not markedly change NEAT1 expression, multiple sgRNAs induced NEAT1 expression (Figure 1C). In particular, sgRNA(MS2)_\#5 exhibited the strongest NEAT1 upregulation ( 4-fold) (Figure 1C). Additionally, multiple sgRNAs including sgRNA(MS2)_Lib\#5, sgRNA(MS2)_Lib\#6, sgRNA(MS2)_Lib\#8, sgRNA(MS2)_\#7, and sgRNA(MS2)_\#8 showed more than 2-fold induction in both NEAT1 5' terminus and NEAT1_2 specific regions (Figure 1C). As a comparison, we used MG132 treatment, which transcriptionally activates NEAT1, leading to 2-fold NEAT1_2 induction under the sgRNA(MS2) empty plasmid transfection condition (Figure 1C) [22]. In addition, several sgRNAs (sgRNA(MS2)_\#5, \#7, and \#8) that strongly activated NEAT1 expression targeted a specific region (-113 to -150) of the NEAT1 promoter with no preference for sense or antisense directions as observed in the SAM-mediated induction of other genes (Figure 1B and 1C) [31]. Transfection efficiency of the SAM system was $\sim 40 \%$ in HAP1 cells, suggesting that actual NEAT1 expression is more strongly activated in the transfected cells (ca. 5-10-fold) than in the cell population as a whole. The ratio of the expression levels of NEAT1 (both NEAT1_1 and NEAT1_2) to NEAT1_2 was similar with or without induction by the SAM system, indicating that the transcriptional activation of NEAT1 does not affect the ratio of production of the two NEAT1 isoforms (Figure S1).

In addition to HAP1 cells, we examined NEAT1 transcriptional activation by the SAM system in human bone osteosarcoma U2OS cells, where NEAT1_2 is lowly expressed and paraspeckles are almost undetectable [18]. We examined the two sgRNAs (sgRNA(MS2)_\#5 
and sgRNA(MS2)_\#7) that strongly activated NEAT1 expression in HAP1 cells. These sgRNAs induced NEAT1 expression in U2OS cells up to 3 -fold compared with the empty vector-transfected cells (Figure $4 \mathrm{~A}$ ). The transfection efficiency was $\sim 50 \%$, suggesting that actual NEAT1 expression is more strongly activated in the transfected cells (ca. 5-6-fold). This suggests that these sgRNAs potently activate NEAT1 expression in two independent human cell lines.

We also examined expression of MALAT1 IncRNA in the NEAT1-induced cells. MALAT1 is another abundant nuclear-retained IncRNA and transcribed from the NEAT1-neighboring gene locus. Previous studies indicated that MALAT1 knockout has an influence on NEAT1 expression levels, suggesting a relationship between the expression of these two IncRNA genes [34, 35]. Overall, MALAT1 IncRNA levels were not strongly changed upon introduction of any sgRNA into HAP1 or U2OS cells (Figure S1A and S1B). However, several sgRNAs such as sgRNA(MS2)_\#5 and sgRNA(MS2)_\#7 slightly induced MALAT1 expression in both HAP1 (1.65- and 1.42-fold induction in \#5 and \#7, respectively) and U2OS (1.33- and 1.65-fold induction in \#5 and \#7, respectively) cells (Figure S1A and S1B), suggesting that NEAT1 induction might lead to MALAT1 upregulation. Additionally, MALAT1 was induced by MG132 treatment (1.85-fold induction) like NEAT1 (Figure S1A and S1B). GAPDH mRNA levels did not change upon introduction of any sgRNA into HAP1 cells (Figure S1A and S1B).

\subsection{SAM system-mediated NEAT1 upregulation facilitates paraspeckle formation}

We next examined whether the NEAT1_2 upregulation leads to paraspeckle formation. We used sgRNAs that effectively activated NEAT1_2 expression levels to more than 2-fold in HAP1 cells (Figure 1C) and monitored paraspeckle formation by smFISH (Figure 2A). The HAP1 cells transfected with the plasmids were identified by GFP signals. We readily recognized the enhanced NEAT1_2 smFISH signals in the transfected cells, and quantified size and sum intensity of the smFISH signals (Figure $2 \mathrm{~B}$ and $2 \mathrm{C}$ ). The signal intensities were correlated to the NEAT1 expression levels quantified by RT-qPCR in Figure 1C, suggesting that NEAT1 induction by the SAM system leads to enlarged paraspeckles. In particular, sgRNA(MS2)_\#5 and \#7 strongly induced paraspeckle formation (Figure 2A-C). In addition, we examined paraspeckle formation upon sgRNA(MS2)_\#5 and \#7 transfections in U2OS cells by smFISH. Although paraspeckles were rarely seen in sgRNA(MS2) empty vector-transfected U2OS cells, significant numbers of paraspeckles with large size and strong fluorescent intensity were observed in sgRNA(MS2)_\#5- and \#7-transfected cells as observed in MG132-treated cells (Figure S2A-C). 
These data suggest that SAM using sgRNA(MS2)_\#5 and \#7 can induce paraspeckle formation even in cells where paraspeckles are normally undetectable.

\subsection{NEAT1 upregulation by the SAM system can induce formation of intact paraspeckles} with ordered core-shell structures

Using SIM, we previously observed core-shell spheroidal structures of paraspeckles, with the 5' and 3' regions of NEAT1_2 located in the shells [17-19]. We next used SIM to examine fine structures of the paraspeckles induced by the SAM system. In $\operatorname{sgRNA}(M S 2)$ empty vector-transfected HAP1 cells, the 5' and 3' regions of NEAT1_2 were located in the shell of the paraspeckles and the core-shell paraspeckles were usually found as separated spheres, consistent with the results of previous studies (Figure 2D) [17, 19]. In the presence of MG132, multiple paraspeckle spheres form and usually adhere to each other [17-19]. The sgRNA(MS2)_\#5 and \#7 introductions also resulted in the formation of multiple paraspeckle spheres, which usually adhered to each other (Figure 2D). These data suggest that the paraspeckles induced by the SAM system have typical core-shell spheroidal structures. 


\section{Discussion}

We demonstrated artificial selective activation of NEAT1 from the endogenous NEAT1 chromosomal locus using the SAM system. Here, we identified multiple sgRNAs that robustly induce NEAT1 expression up to $3-4$-fold in two human cell lines by screening 25 sgRNAs targeting the NEAT1 promoter. Our confocal and super-resolution microscopic analyses also validated that the NEAT1 induction facilitates formation of paraspeckles possessing known core-shell structures. The identified sgRNAs (e.g., sgRNA(MS2)_\#5 and \#7) induced NEAT1 expression and paraspeckle formation regardless of different steady-state NEAT1 levels. As in U2OS cells, NEAT1 is poorly expressed and paraspeckles are almost undetectable in multiple human cell lines [18]. Thus, our selected sgRNAs and SAM system could be used to investigate the effects of NEAT1 overexpression in such cell lines. MG132 can transcriptionally activate NEAT1, but it also causes pleiotropic effects including proteostatic stress to cells [22]. Therefore, this SAM-mediated selective NEAT1 upregulation method enables us to purely investigate the effects of NEAT1 expression from the endogenous locus on cellular functions, leading to greater understanding of the biological roles of NEAT1 IncRNA in various cell lines.

Among multiple sgRNAs that strongly activate NEAT1 expression, several of our designed sgRNAs produced stronger NEAT1 induction than pre-designed ones. By using several validated sgRNAs, it would be possible to obtain more reliable data on NEAT1 functions by excluding the possibilities of off-target effects. Combinational use of active sgRNAs targeting different NEAT1 promoter regions might achieve further enhancement of NEAT1 expression as described previously [36]. Interestingly, two sgRNAs strongly activating NEAT1 expression commonly induced MALAT1 in HAP1 and U2OS cells. Previous reports show that MALAT1 dysfunctions affect NEAT1 expression levels $[34,35]$. The recent CHART analysis against NEAT1 revealed that NEAT1 targets the MALAT1 promoter [25]. Interestingly, we showed that both NEAT1 and MALAT1 are induced by MG132 treatment, and SAM-mediated NEAT1 induction by several sgRNAs was accompanied by MALAT1 upregulation, although an exception (sgRNA(MS2)_\#8) was observed in HAP1 cells. These results raise the possibility that NEAT1 and MALAT1 expression is co-regulated. Further careful analyses will be required to confirm regulation of MALAT1 expression by NEAT1.

NEAT1 is one of the most abundant, well-studied arcRNAs and thus is an ideal model for arcRNAs that trigger phase separation upon their expression. This SAM-mediated NEAT1 induction method would be useful for various downstream analyses such as RNA-seq, ChIRP (chromatin isolation by RNA purification), and Hi-C. By combining these technologies, we will be 
able to investigate the molecular mechanisms and roles of NEAT1 in gene expression, 3D genome organization, and nuclear architecture. Furthermore, it might be possible to investigate the relationship between phase separation and 3D genome organization $[10,19,37]$. Thus, this method would contribute to understanding of the molecular mechanism underlying IncRNA-based nuclear architecture. 


\section{References}

[1] I. Ulitsky, Evolution to the rescue: using comparative genomics to understand long non-coding RNAs, Nat Rev Genet, 17 (2016) 601-614.

[2] J.J. Quinn, H.Y. Chang, Unique features of long non-coding RNA biogenesis and function, Nat Rev Genet, 17 (2016) 47-62.

[3] M. Guttman, J.L. Rinn, Modular regulatory principles of large non-coding RNAs, Nature, 482 (2012) 339-346.

[4] T. Hirose, Y. Mishima, Y. Tomari, Elements and machinery of non-coding RNAs: toward their taxonomy, EMBO Rep, 15 (2014) 489-507.

[5] P.J. Batista, H.Y. Chang, Long noncoding RNAs: cellular address codes in development and disease, Cell, 152 (2013) 1298-1307.

[6] T. Chujo, T. Yamazaki, T. Hirose, Architectural RNAs (arcRNAs): A class of long noncoding RNAs that function as the scaffold of nuclear bodies, Biochim Biophys Acta, 1859 (2016) 139-146.

[7] Y.T. Sasaki, T. Ideue, M. Sano, T. Mituyama, T. Hirose, MENepsilon/beta noncoding RNAs are essential for structural integrity of nuclear paraspeckles, Proc Natl Acad Sci U S A, 106 (2009) 2525-2530.

[8] C.M. Clemson, J.N. Hutchinson, S.A. Sara, A.W. Ensminger, A.H. Fox, A. Chess, J.B. Lawrence, An architectural role for a nuclear noncoding RNA: NEAT1 RNA is essential for the structure of paraspeckles, Mol Cell, 33 (2009) 717-726.

[9] H. Sunwoo, M.E. Dinger, J.E. Wilusz, P.P. Amaral, J.S. Mattick, D.L. Spector, MEN epsilon/beta nuclear-retained non-coding RNAs are up-regulated upon muscle differentiation and are essential components of paraspeckles, Genome Res, 19 (2009) 347-359.

[10] A.H. Fox, S. Nakagawa, T. Hirose, C.S. Bond, Paraspeckles: Where Long Noncoding RNA Meets Phase Separation, Trends Biochem Sci, 43 (2018) 124-135.

[11] N. Visa, F. Puvion-Dutilleul, J.P. Bachellerie, E. Puvion, Intranuclear distribution of U1 and U2 snRNAs visualized by high resolution in situ hybridization: revelation of a novel compartment containing U1 but not U2 snRNA in HeLa cells, Eur J Cell Biol, 60 (1993) 308-321.

[12] A.H. Fox, Y.W. Lam, A.K. Leung, C.E. Lyon, J. Andersen, M. Mann, A.I. Lamond, Paraspeckles: a novel nuclear domain, Curr Biol, 12 (2002) 13-25.

[13] T. Naganuma, S. Nakagawa, A. Tanigawa, Y.F. Sasaki, N. Goshima, T. Hirose, Alternative 3'-end processing of long noncoding RNA initiates construction of nuclear paraspeckles, EMBO J, 31 (2012) 4020-4034. 
[14] K.W. Fong, Y. Li, W. Wang, W. Ma, K. Li, R.Z. Qi, D. Liu, Z. Songyang, J. Chen, Whole-genome screening identifies proteins localized to distinct nuclear bodies, J Cell Biol, 203 (2013) 149-164.

[15] T. Yamazaki, T. Hirose, The building process of the functional paraspeckle with long non-coding RNAs, Front Biosci (Elite Ed), 7 (2015) 1-41.

[16] S. Souquere, G. Beauclair, F. Harper, A. Fox, G. Pierron, Highly ordered spatial organization of the structural long noncoding NEAT1 RNAs within paraspeckle nuclear bodies, Mol Biol Cell, 21 (2010) 4020-4027.

[17] J.A. West, M. Mito, S. Kurosaka, T. Takumi, C. Tanegashima, T. Chujo, K. Yanaka, R.E. Kingston, T. Hirose, C. Bond, A. Fox, S. Nakagawa, Structural, super-resolution microscopy analysis of paraspeckle nuclear body organization, J Cell Biol, 214 (2016) 817-830.

[18] T. Chujo, T. Yamazaki, T. Kawaguchi, S. Kurosaka, T. Takumi, S. Nakagawa, T. Hirose, Unusual semi-extractability as a hallmark of nuclear body-associated architectural noncoding RNAs, EMBO J, 36 (2017) 1447-1462.

[19] T. Yamazaki, S. Souquere, T. Chujo, S. Kobelke, Y.S. Chong, A.H. Fox, C.S. Bond, S. Nakagawa, G. Pierron, T. Hirose, Functional Domains of NEAT1 Architectural lncRNA Induce Paraspeckle Assembly through Phase Separation, Mol Cell, 70 (2018) 1038-1053 e1037.

[20] S. Hennig, G. Kong, T. Mannen, A. Sadowska, S. Kobelke, A. Blythe, G.J. Knott, K.S. Iyer, D. Ho, E.A. Newcombe, K. Hosoki, N. Goshima, T. Kawaguchi, D. Hatters, L. Trinkle-Mulcahy, T. Hirose, C.S. Bond, A.H. Fox, Prion-like domains in RNA binding proteins are essential for building subnuclear paraspeckles, J Cell Biol, 210 (2015) 529-539.

[21] Y.S. Mao, H. Sunwoo, B. Zhang, D.L. Spector, Direct visualization of the co-transcriptional assembly of a nuclear body by noncoding RNAs, Nat Cell Biol, 13 (2011) 95-101.

[22] T. Hirose, G. Virnicchi, A. Tanigawa, T. Naganuma, R. Li, H. Kimura, T. Yokoi, S. Nakagawa, M. Benard, A.H. Fox, G. Pierron, NEAT1 long noncoding RNA regulates transcription via protein sequestration within subnuclear bodies, Mol Biol Cell, 25 (2014) 169-183.

[23] L.L. Chen, G.G. Carmichael, Altered nuclear retention of mRNAs containing inverted repeats in human embryonic stem cells: functional role of a nuclear noncoding RNA, Mol Cell, 35 (2009) 467-478. [24] K. Imamura, N. Imamachi, G. Akizuki, M. Kumakura, A. Kawaguchi, K. Nagata, A. Kato, Y. Kawaguchi, H. Sato, M. Yoneda, C. Kai, T. Yada, Y. Suzuki, T. Yamada, T. Ozawa, K. Kaneki, T. Inoue, M. Kobayashi, T. Kodama, Y. Wada, K. Sekimizu, N. Akimitsu, Long noncoding RNA NEAT1-dependent SFPQ relocation from promoter region to paraspeckle mediates IL8 expression upon immune stimuli, Mol Cell, 53 (2014) 393-406. 
[25] J.A. West, C.P. Davis, H. Sunwoo, M.D. Simon, R.I. Sadreyev, P.I. Wang, M.Y. Tolstorukov, R.E. Kingston, The long noncoding RNAs NEAT1 and MALAT1 bind active chromatin sites, Mol Cell, 55 (2014) 791-802.

[26] S. Nakagawa, M. Shimada, K. Yanaka, M. Mito, T. Arai, E. Takahashi, Y. Fujita, T. Fujimori, L. Standaert, J.C. Marine, T. Hirose, The lncRNA Neat1 is required for corpus luteum formation and the establishment of pregnancy in a subpopulation of mice, Development, 141 (2014) 4618-4627.

[27] C. Adriaens, L. Standaert, J. Barra, M. Latil, A. Verfaillie, P. Kalev, B. Boeckx, P.W. Wijnhoven, E. Radaelli, W. Vermi, E. Leucci, G. Lapouge, B. Beck, J. van den Oord, S. Nakagawa, T. Hirose, A.A. Sablina, D. Lambrechts, S. Aerts, C. Blanpain, J.C. Marine, p53 induces formation of NEAT1 lncRNA-containing paraspeckles that modulate replication stress response and chemosensitivity, Nat Med, 22 (2016) 861-868.

[28] S.S. Mello, C. Sinow, N. Raj, P.K. Mazur, K. Bieging-Rolett, D.K. Broz, J.F.C. Imam, H. Vogel, L.D. Wood, J. Sage, T. Hirose, S. Nakagawa, J. Rinn, L.D. Attardi, Neat1 is a p53-inducible lincRNA essential for transformation suppression, Genes Dev, 31 (2017) 1095-1108.

[29] E. Rheinbay, P. Parasuraman, J. Grimsby, G. Tiao, J.M. Engreitz, J. Kim, M.S. Lawrence, A. Taylor-Weiner, S. Rodriguez-Cuevas, M. Rosenberg, J. Hess, C. Stewart, Y.E. Maruvka, P. Stojanov, M.L. Cortes, S. Seepo, C. Cibulskis, A. Tracy, T.J. Pugh, J. Lee, Z. Zheng, L.W. Ellisen, A.J. Iafrate, J.S. Boehm, S.B. Gabriel, M. Meyerson, T.R. Golub, J. Baselga, A. Hidalgo-Miranda, T. Shioda, A. Bernards, E.S. Lander, G. Getz, Recurrent and functional regulatory mutations in breast cancer, Nature, 547 (2017) $55-60$.

[30] O. Shalem, N.E. Sanjana, F. Zhang, High-throughput functional genomics using CRISPR-Cas9, Nat Rev Genet, 16 (2015) 299-311.

[31] S. Konermann, M.D. Brigham, A.E. Trevino, J. Joung, O.O. Abudayyeh, C. Barcena, P.D. Hsu, N. Habib, J.S. Gootenberg, H. Nishimasu, O. Nureki, F. Zhang, Genome-scale transcriptional activation by an engineered CRISPR-Cas9 complex, Nature, 517 (2015) 583-588.

[32] J. Joung, S. Konermann, J.S. Gootenberg, O.O. Abudayyeh, R.J. Platt, M.D. Brigham, N.E. Sanjana, F. Zhang, Genome-scale CRISPR-Cas9 knockout and transcriptional activation screening, Nat Protoc, 12 (2017) 828-863.

[33] F.A. Ran, P.D. Hsu, J. Wright, V. Agarwala, D.A. Scott, F. Zhang, Genome engineering using the CRISPR-Cas9 system, Nat Protoc, 8 (2013) 2281-2308.

[34] S. Nakagawa, J.Y. Ip, G. Shioi, V. Tripathi, X. Zong, T. Hirose, K.V. Prasanth, Malat1 is not an essential component of nuclear speckles in mice, RNA, 18 (2012) 1487-1499. 
[35] B. Zhang, G. Arun, Y.S. Mao, Z. Lazar, G. Hung, G. Bhattacharjee, X. Xiao, C.J. Booth, J. Wu, C. Zhang, D.L. Spector, The lncRNA Malat1 is dispensable for mouse development but its transcription plays a cis-regulatory role in the adult, Cell Rep, 2 (2012) 111-123.

[36] A. Chavez, M. Tuttle, B.W. Pruitt, B. Ewen-Campen, R. Chari, D. Ter-Ovanesyan, S.J. Haque, R.J. Cecchi, E.J.K. Kowal, J. Buchthal, B.E. Housden, N. Perrimon, J.J. Collins, G. Church, Comparison of Cas9 activators in multiple species, Nat Methods, 13 (2016) 563-567.

[37] Y. Shin, C.P. Brangwynne, Liquid phase condensation in cell physiology and disease, Science, 357 (2017). 


\section{Acknowledgments}

The authors thank the members of the Hirose laboratory for discussion. This work was supported by JSPS KAKENHI Grant Numbers 15K18474, 17K15058, 26113002, 17H03630, and $17 \mathrm{~K} 19335$.

\section{Author contributions}

TY and TH conceived and designed the study. TY, CF, AT, and AK conducted the experiments.

TY and TH wrote the manuscript.

\section{Additional information}

Competing Interests: The authors declare no competing interests. 
Table 1. sgRNA list

\begin{tabular}{|c|c|c|c|}
\hline Name & Sequence ( $5^{\prime}$ to $\left.3^{\prime}\right)$ & Position (Sense/Antisense) & Score \\
\hline Lib\#1 & GCCAGAGAAACCGCCTGTTG & $-644 /-625(S)$ & 83 \\
\hline Lib\#2 & GGGAGGCGCGCGTGACTTTC & $-506 /-487$ (AS) & 87 \\
\hline Lib\#3 & GCGCCCTCTTGAGTGGGCGA & $-364 /-345(A S)$ & 86 \\
\hline Lib\#4 & TTTCTTCCCGGATGTTGTTG & $-289 / 270(A S)$ & 73 \\
\hline Lib\#5 & TTTGGGAGGCGAATGCCATG & $-254 / 235$ (AS) & 73 \\
\hline Lib\#6 & AGCACTGTTAAAGAGAAGCG & $-202 /-183(S)$ & 74 \\
\hline Lib\#7 & CGGGCGGTGCATGGTCTCCC & $-148 /-129$ (AS) & 85 \\
\hline Lib\#8 & AGTCTCTCCGGGCAGGGTCG & $-126 /-107(S)$ & 74 \\
\hline Lib\#9 & GTCCCGTTGAGCAATGACCC & $-70 /-51(S)$ & 87 \\
\hline Lib\#10 & ATAGCCCTCAGCCGCGTCAC & $-48 /-29$ (AS) & 94 \\
\hline$\# 1$ & GATACACTGGGGTCCTTGCG & $-180 /-161(S)$ & 87 \\
\hline \#2 & ATACACTGGGGTCCTTGCGT & $-179 /-160(S)$ & 90 \\
\hline \#3 & TCCCAGGCGGCCCCCACGCA & $-164 /-145(A S)$ & 64 \\
\hline$\# 4$ & GCGGTGCATGGTCTCCCAGG & $-151 /-132(A S)$ & 63 \\
\hline \#5 & CTGGGAGACCATGCACCGCC & $-150 /-131(S)$ & 83 \\
\hline \#6 & TGGGAGACCATGCACCGCCC & $-149 /-130(S)$ & 86 \\
\hline \#7 & AGAGACTCCCGGGCGGTGCA & $-139 /-120(\mathrm{AS})$ & 79 \\
\hline \#8 & GCACCGCCCGGGAGTCTCTC & $-138 /-119(\mathrm{~S})$ & 78 \\
\hline$\# 9$ & CCCGGGAGTCTCTCCGGGCA & $-132 /-113(\mathrm{~S})$ & 77 \\
\hline$\# 10$ & TGCCCGGAGAGACTCCCGGG & $-132 /-113(A S)$ & 72 \\
\hline$\# 11$ & GGGAGGGACTTTTTTGCCGG & $-106 /-87(S)$ & 88 \\
\hline$\# 12$ & GACTTTTTTGCCGGTGGCCG & $-100 /-81(S)$ & 91 \\
\hline$\# 13$ & CACCGGGGTCATTGCTCAAC & $-65 /-46$ (AS) & 90 \\
\hline$\# 14$ & GAGCAATGACCCCGGTGACG & $-62 /-43(S)$ & 92 \\
\hline \#15 & GACCCCGGTGACGCGGCTGA & $-55 /-36(S)$ & 92 \\
\hline
\end{tabular}


Figure 1. Transcriptional activation of NEAT1 by the SAM system. (A) The strategy for transcriptional activation of the NEAT1 IncRNA gene. Three plasmids encoding sgRNA(MS2), dCas9-VP64, and MS2-P65-HSF1 were transfected into human cells and cultured for 48 hours. The cells were then subjected to further downstream analyses (e.g., RT-qPCR and FISH). (B) Positions of sgRNAs targeting the NEAT1 promoter regions. Magenta bars indicate pre-designed sgRNAs from the CRISPRa library, and blue bars indicate newly designed sgRNAs. Direction of sgRNAs is shown as sense (upper) or antisense (lower). (C and D) RT-qPCR analysis of NEAT1 expression upon sgRNA transfection with the SAM system in human HAP1 cells (C) and U2OS cells (D). NEAT1 primers (dark blue) targeting both NEAT1_1 and NEAT1_2, and NEAT1_2 specific primers (light blue), were used to quantify NEAT1 levels. NEAT1 levels in HAP1 cells transfected with sgRNA(MS2) empty plasmids, dCas9-VP64, and MS2-P65-HSF1 were used as the standard (Empty, value $=1)$. Data are represented as mean $\pm S D(n=3)$.

Figure 2. NEAT1 upregulation by the SAM system leads to paraspeckle formation and ordered paraspeckle assembly. (A) Representative images of HAP1 cells transfected with sgRNAs and SAM system plasmids. GFP-positive cells represent the cells successfully transfected with SAM system plasmids. Paraspeckles (NEAT1 foci) were visualized with smFISH. Nuclei were stained with DAPI. Scale bar: $10 \mu \mathrm{m}$. (B) Quantification of area of paraspeckles per GFP-positive HAP1 cell. Kruskal-Wallis test with Dunn's multiple comparison test was used. ${ }^{\star * * *}: P<0.0001,{ }^{* \star *}: P$ $<0.001,{ }^{* *}: P<0.01,{ }^{*}: P<0.05$.) (C) Quantification of sum intensity of paraspeckles per GFP-positive HAP1 cell. Each box plot shows median (inside line) and 10-90 percentiles (box bottom to top). Kruskal-Wallis test with Dunn's multiple comparison test was used. ${ }^{* \star * *}: P<$ $0.0001,{ }^{* *}: P<0.001,{ }^{* *}: P<0.01,{ }^{*}: P<0.05$.) (D) Representative SIM images of NEAT1 FISH in HAP1 cells on indicated conditions. NEAT1 probes targeting the NEAT1 5 ' region and NEAT1_2 3' region (magenta) were used to visualize shells of the paraspeckles. Paraspeckles formed in the GFP-positive cells were observed. Scale bar: $500 \mathrm{~nm}$. 
Table S1. Primers used in this study

\begin{tabular}{l|ll} 
Primers & Forward (5' to $3^{\prime}$ ) & Reverse (5' to 3') \\
\hline NEAT1 & CAATTACTGTCGTTGGGATTTAGAGTG & TTCTTACCATACAGAGCAACATACCAG \\
\hline NEAT1_2 & TGTGTGTGTAAAAGAGAGAAGTTGTGG & AGAGGCTCAGAGAGGACTGTAACCTG \\
\hline MALAT1 & GATAATCAGACCACCACAGGTTTACAG & AAAGAGTAACTACCAGCCATTTCTCCA \\
\hline 18S rRNA & TTTAAGTTTCAGCTTTGCAACCATACT & ATTAACAAGAACGAAAGTCGGAGGT \\
\hline GAPDH & ATGAGAAGTATGACAACAGCCTCAAGAT & ATGAGTCCTTCCACGATACCAAAGTT
\end{tabular}


Figure S1. The ratio of NEAT1 isoforms, and expression of MALAT1 and GAPDH upon the SAM transfections. (A, B) The ratio of NEAT1 (NEAT1_1 and NEAT1_2) and NEAT1_2 in the cells shown in Figure 1C (A) and 1D (B). (C, D) MALAT1 and GAPDH expression levels in the cells shown in Figure 1C (C) and 1D (D). RT-qPCR analysis of MALAT1 (dark blue) and GAPDH (light blue) expression in HAP1 (C) and U2OS cells (D). RNA levels in Empty were used as the standard $($ Empty, value $=1)$. Data are represented as mean $\pm \operatorname{SD}(n=3)$.

Figure S2. NEAT1 upregulation by the SAM system leads to paraspeckle elongation in the U2OS cell line. (A) Representative images of U2OS cells transfected with sgRNAs and SAM system plasmids. GFP-positive cells indicate cells successfully transfected with SAM system plasmids. Paraspeckles (NEAT1 foci) were visualized with smFISH. Nuclei were stained with DAPI. Scale bar: $10 \mu \mathrm{m}$. (B) Quantification of area of paraspeckles per GFP-positive cell in U2OS cells. Kruskal-Wallis test with Dunn's multiple comparison test was used. ( ${ }^{* * *}: P<$ 0.0001.) (C) Quantification of sum intensity of paraspeckles per GFP-positive cell in U2OS cells. Each box plot shows median (inside line) and 10-90 percentiles (box bottom to top). Kruskal-Wallis test with Dunn's multiple comparison test was used. ${ }^{\star * \star *}: P<0.0001$.) 
A

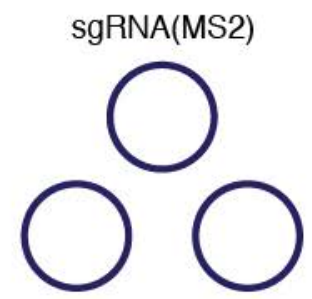

\section{Plasmid}

Transfection
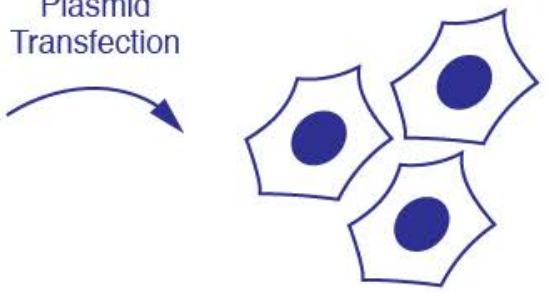

Downstream analyses

(RT-qPCR \& FISH)

dCas9-VP64 MS2-p65-HSF1

B

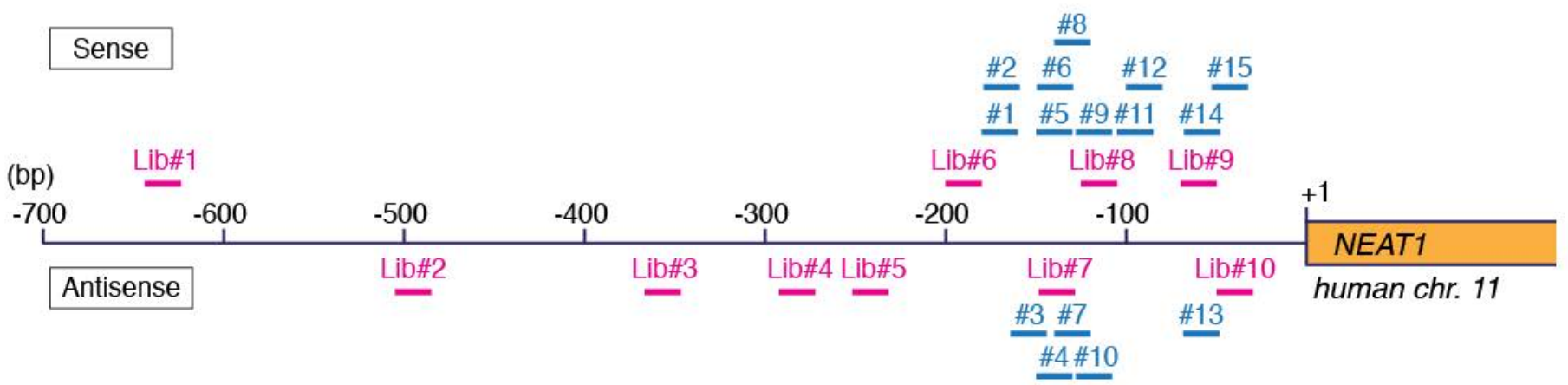

C
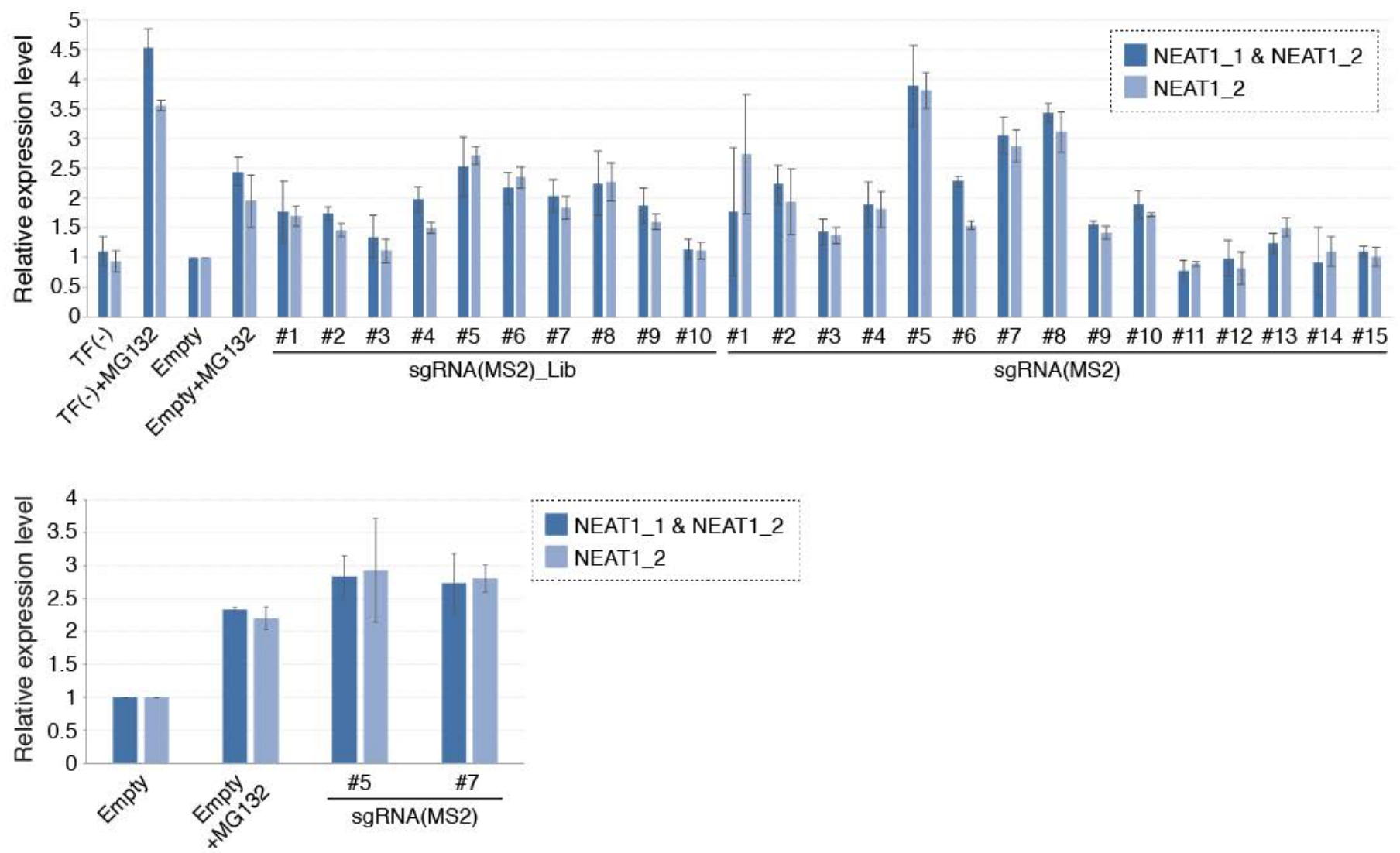
A

sgRNA(MS2)

Empty

Lib_\#5

Lib_\#6

Lib_\#8

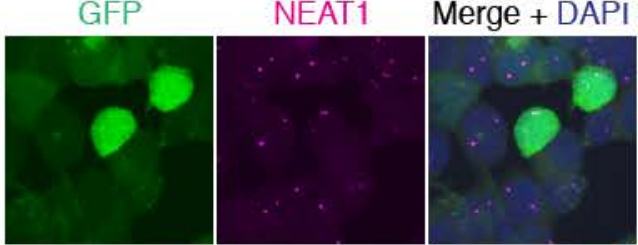

Lib_\#10
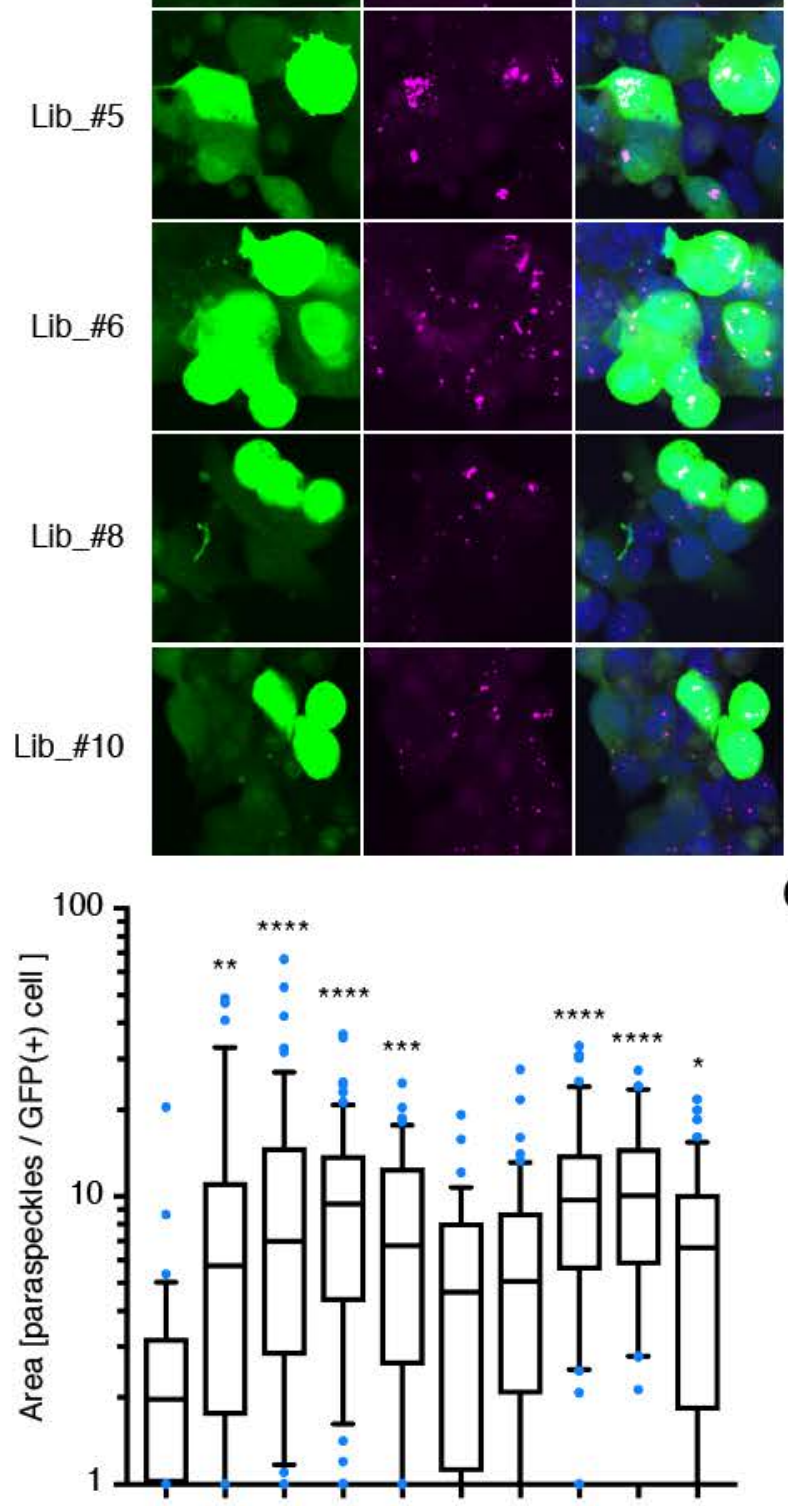

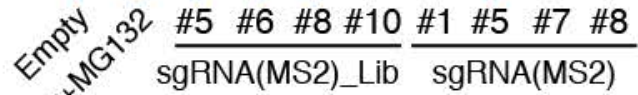
$\sin ^{x+x^{x}}$

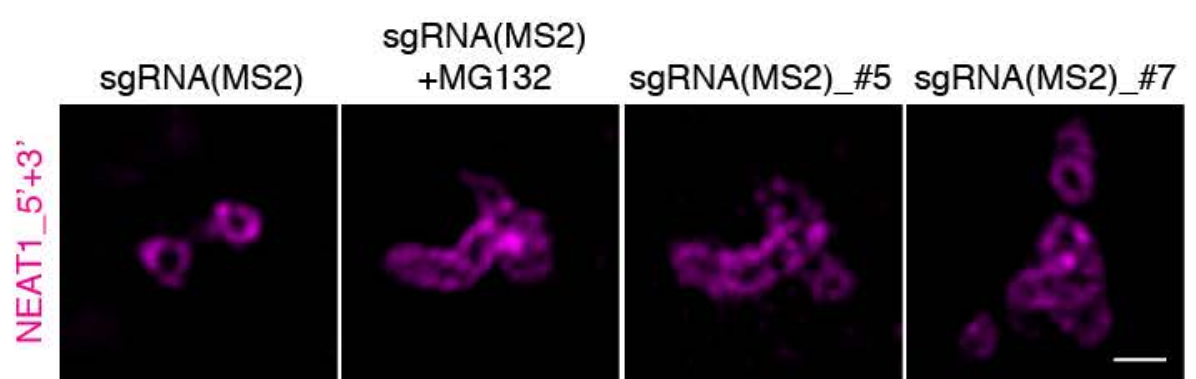

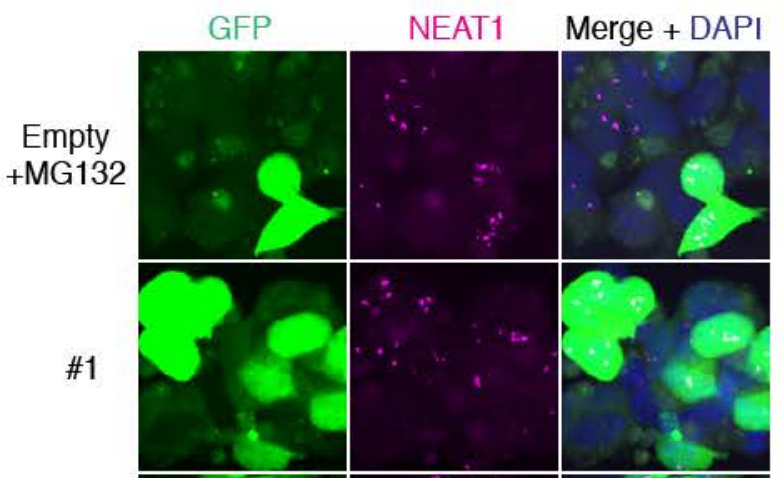

\#5

\#7

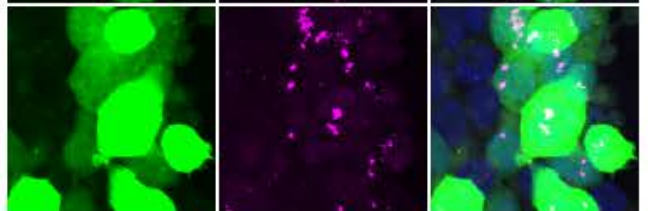

\#8

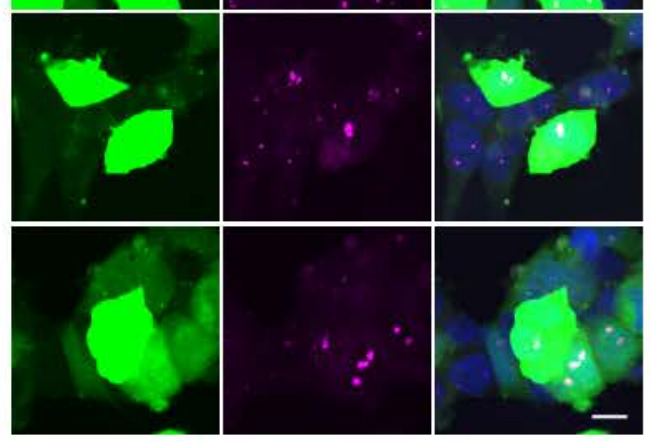

C

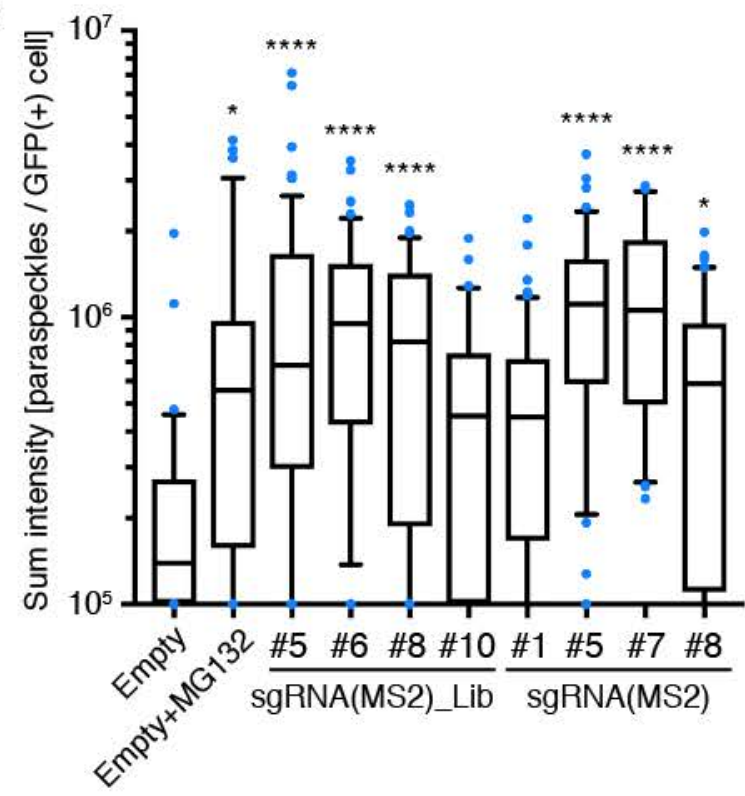


A

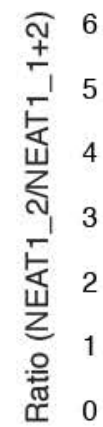

若 से $\left\langle x^{x^{x}}\right.$

B
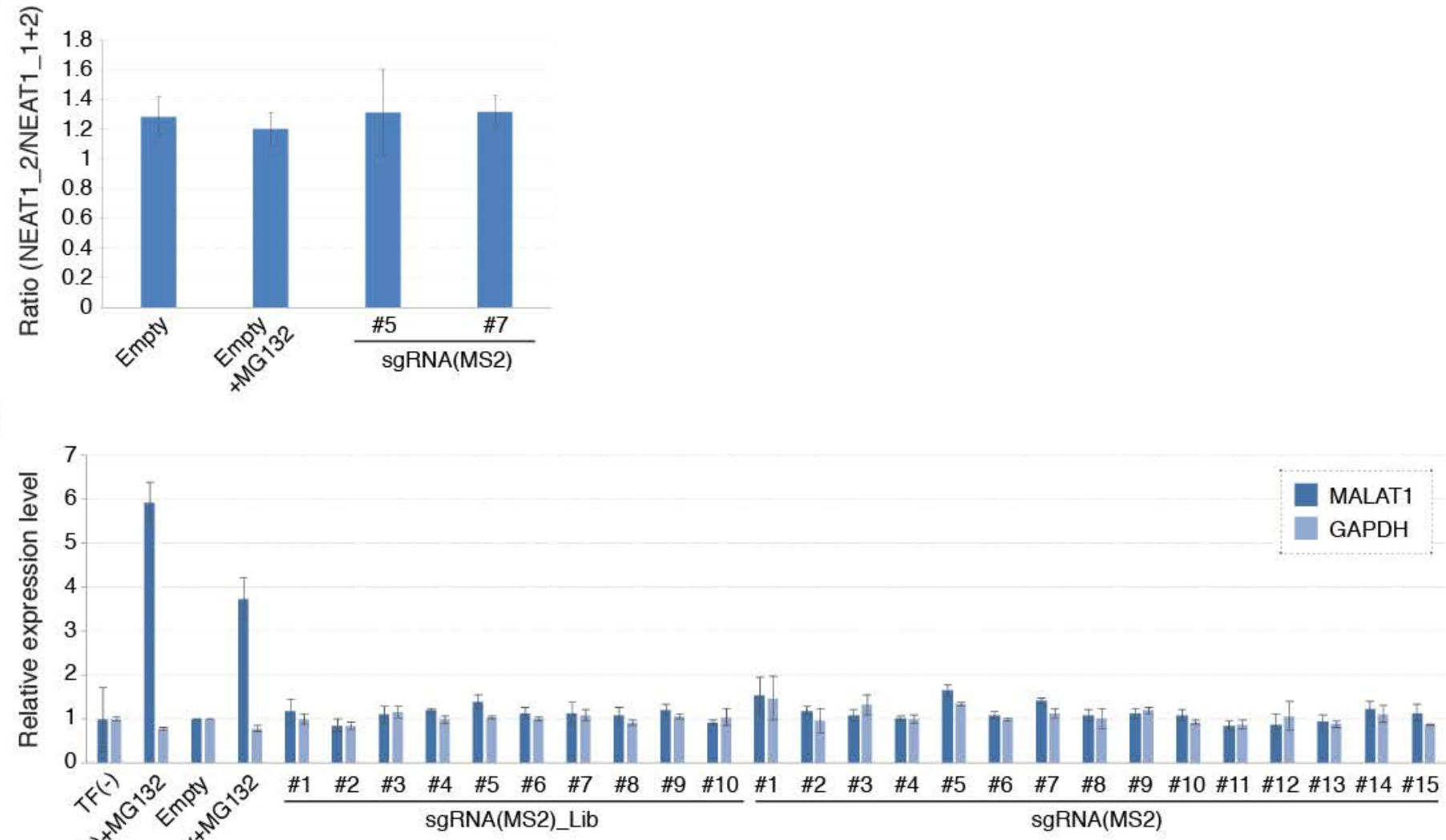
음 


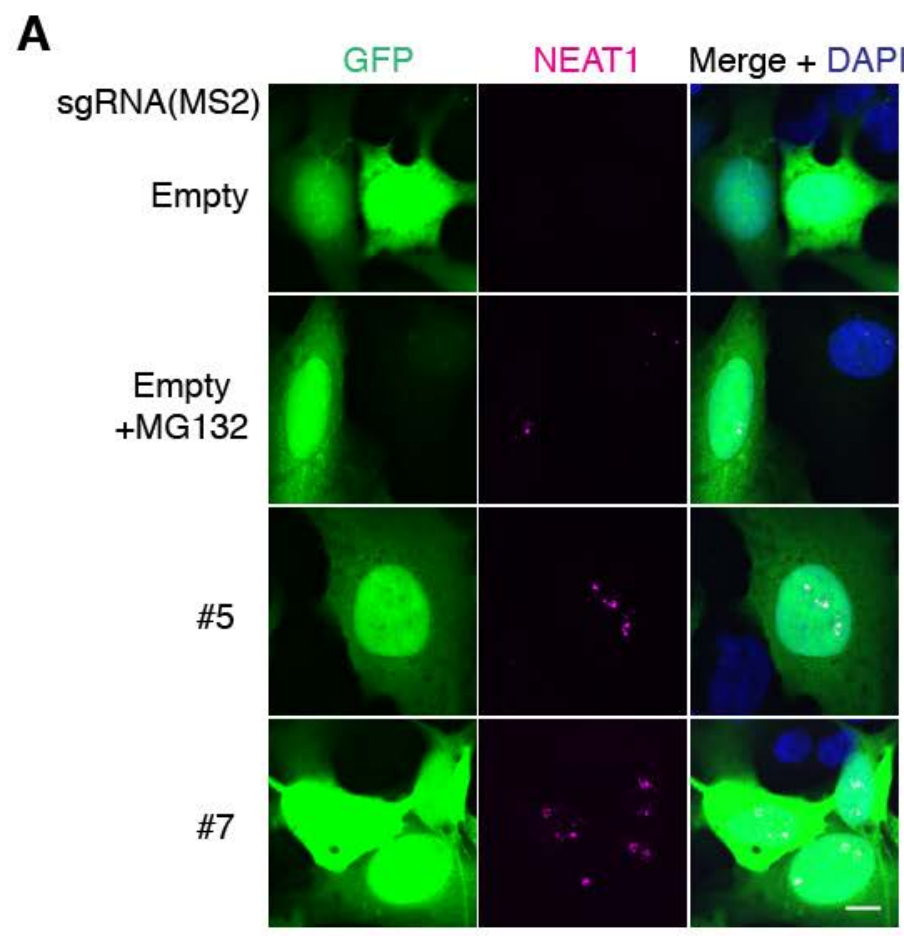

B

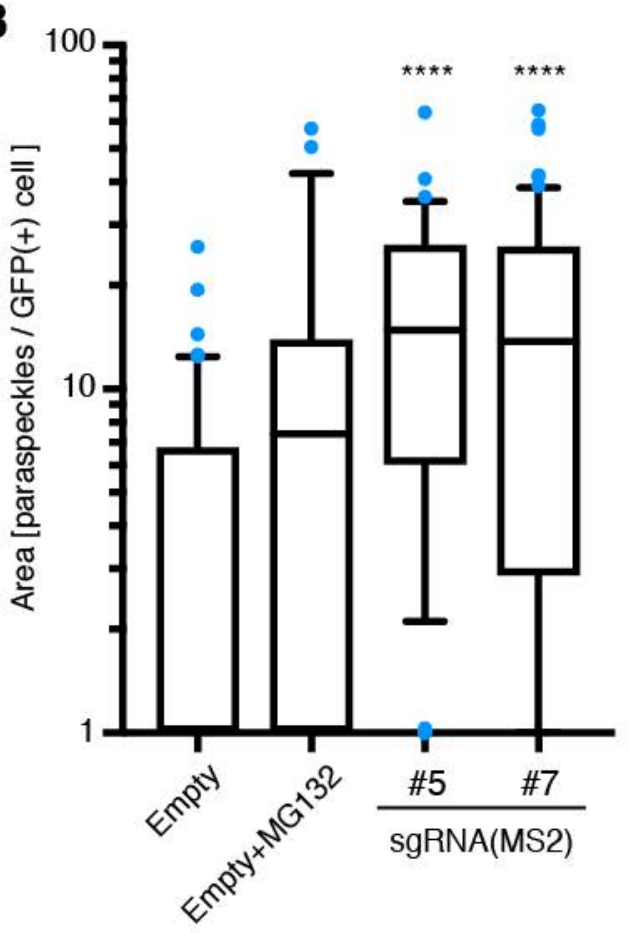

C

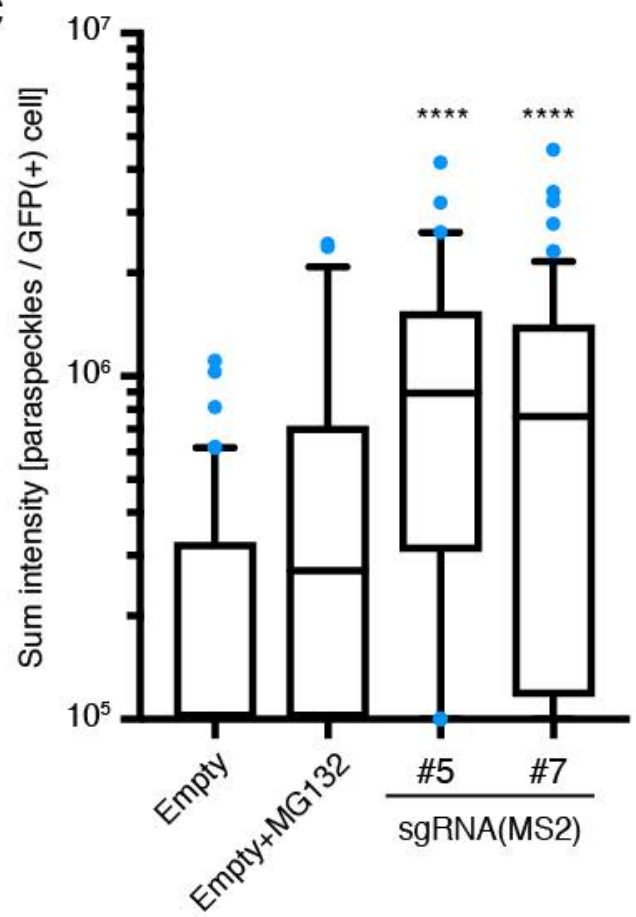

